\title{
Health care resource use among patients with advanced non-small cell lung cancer: the PIVOTAL retrospective observational study
}

Dae Ho Lee ${ }^{1}$, Hiroshi Isobe ${ }^{2}$, Hubert Wirtz 3 , Sabina Bandeira Aleixo ${ }^{4}$, Phillip Parente ${ }^{5}$, Filippo de Marinis ${ }^{6}$, Min Huang ${ }^{7}$, Ashwini Arunachalam ${ }^{8^{*}}$ D, Smita Kothari ${ }^{8}$, Xiting $\mathrm{CaO}^{8}$, Nello Donnini ${ }^{9}$, Ann-Marie Woodgate ${ }^{10}$ and Javier de Castro ${ }^{11}$

\begin{abstract}
Background: Data are scarce regarding real-world health care resource use (HCRU) for non-small cell lung cancer (NSCLC). An understanding of current clinical practices and HCRU is needed to provide a benchmark for rapidly evolving NSCLC management recommendations and therapeutic options. The objective of this study was to describe real-world HCRU for patients with advanced NSCLC.

Methods: This multinational, retrospective chart review study was conducted at academic and community oncology sites in Italy, Spain, Germany, Australia, Japan, South Korea, Taiwan, and Brazil. Deidentified data were drawn from medical records of 1440 adults ( $\geq 18$ years old) who initiated systemic therapy (2011 to mid-2013) for a new, confirmed diagnosis of advanced or metastatic (stage IIIB or IV) NSCLC. We summarized HCRU associated with first and subsequent lines of systemic therapy for advanced/metastatic NSCLC.

Results: The proportion of patients who were hospitalized at least once varied by country from $24 \%$ in Italy to $81 \%$ in Japan during first-line therapy and from 22\% in Italy to 84\% in Japan during second-line therapy; overall hospitalization frequency was 2.5-11.1 per 100 patient-weeks, depending on country. Emergency visit frequency also varied among countries (overall from 0.3-5.9 per 100 patient-weeks), increasing consistently from first- through third-line therapy in each country. The outpatient setting was the most common setting of resource use. Most patients in the study had multiple outpatient visits in association with each line of therapy (overall from 21.1 to 59.0 outpatient visits per 100 patient-weeks, depending on country). The use of health care resources showed no regular pattern associated with results of tests for activating mutations of the epidermal growth factor receptor (EGFR) gene or anaplastic lymphoma kinase (ALK) gene rearrangements.

Conclusions: HCRU varied across countries. These findings suggest differing approaches to the clinical management of advanced NSCLC among the eight countries. Comparative findings and an understanding of country-specific clinical practices can help to identify areas of need and guide future resource allocation for patients with advanced NSCLC. Further studies evaluating the costs associated with resource use are warranted.
\end{abstract}

Keywords: Emergency visits, Health care resource use, Hospitalizations, Non-small cell lung cancer, NSCLC, Outpatient visits

\footnotetext{
* Correspondence: ashwini.arunachalam@merck.com

${ }^{8}$ Center for Observational and Real World Evidence (CORE), Merck \& Co., Inc.,

2000 Galloping Hill Road, Kenilworth, NJ 07033, USA

Full list of author information is available at the end of the article
} 


\section{Background}

Lung cancer was responsible for 1.6 million deaths globally in 2012 [1]. Non-small cell lung cancer (NSCLC) is the most common form, accounting for $80-85 \%$ of all cases of lung cancer [1-3]. Risk factors for developing NSCLC include tobacco smoking (the number one risk factor) and exposure to second-hand tobacco smoke, airborne carcinogens, radon gas, and cooking fumes [1, 4]. The diagnosis of NSCLC is often, indeed in up to $65 \%$ of cases, made in advanced stages when the tumor(s) are nonresectable because of local infiltration or distant metastasis (stages IIIB and IV) $[5,6]$. Until recently, the recommended systemic therapy for patients with advanced (nonresectable) NSCLC has been platinum-based chemotherapy in first-line, followed by single agents, such as docetaxel or pemetrexed, for patients who fail to respond or who experience disease progression after chemotherapy [6]. Even with therapy, the 5-year survival rates for patients with advanced NSCLC are $5 \%$ or lower [4].

Fortunately the list of treatment options for advanced NSCLC has expanded rapidly in the past 15 years, with continued optimistic outlook for expansion, because of two major advances: the discovery of molecular biomarkers that identify improved response to targeted agents and the development of immunotherapies, which assist patients' immune response in attacking their cancer [6-8]. Currently the two most commonly diagnosed targetable biomarkers, each of which are accompanied by improved potential for response to specific tyrosine kinase inhibitors (TKIs), are activating mutations of the epidermal growth factor receptor (EGFR) gene and anaplastic lymphoma kinase $(A L K)$ gene rearrangements. Immunotherapies recently approved for treating advanced NSCLC include the programmed death-1 (PD1) receptor inhibitors, nivolumab and pembrolizumab [9]. The place in therapy for these newer agents remains an area of active study.

Patients with NSCLC usually require high levels of health care resource use (HCRU) that includes diagnostic testing, treatments, frequent office visits, potential hospitalizations, as well as supportive care at home, in hospital, and in hospice. However, data are scarce regarding the real-world utilization of health care resources for NSCLC [10]. Clinical trial data have limited utility for assessing real-world HCRU both because key information, such as that regarding hospitalizations, may not be collected and because patients enrolled in clinical trials are not representative of those treated in realworld clinical practice [11]. The eligibility criteria for NSCLC clinical trials typically exclude patients with poor performance status, comorbidities, untreated brain metastases, or insufficient biopsy tissue to fully characterize their cancer. Therefore real-world data are important to understand HCRU in routine clinical practice and, as important, for use in economic modeling to support decision-making by providers and payers [12].

The PIvOTAL study (Global treatment Patterns, resource utilization and bIOmarker Testing of Advanced non-small cell Lung cancer) was a retrospective observational study designed to address the scarcity of realworld data regarding the treatment of patients with advanced/metastatic (Stage IIIB/IV) NSCLC in nine countries in different regions of the world. The primary objectives of the study were to examine treatment patterns, biopsy and predictive biomarker testing patterns, and associated HCRU for patients with advanced NSCLC. Here we report the HCRU findings from eight countries in the PIvOTAL study. The treatment pattern data for these eight countries have been previously published $[13,14]$, and the findings from Canada are being considered for future publication.

\section{Methods}

\section{Data source and patients}

This multinational, retrospective observational study was conducted at both academic and community oncology sites in Italy, Spain, Germany, Australia, Japan, South Korea, Taiwan, and Brazil. Detailed study methods have been published [13]. Electronic case report forms (eCRFs) were used to collect de-identified data abstracted from medical records of patients with stage IIIB/IV NSCLC. A single individual at each site was charged with data abstraction to maximize consistency, working under the supervision of a lead site investigator who was responsible for data review.

Eligible patients for study were adults ( $\geq 18$ years old) who received systemic therapy for a new diagnosis between January 1, 2011, and July 1, 2013, of histopathologically confirmed advanced/metastatic NSCLC (stage IIIB or IV, confirmed by tissue biopsy or cytology). In addition, eligible patients had to have complete medical records from the time of diagnosis to the end of the study, or death, whichever occurred first. Patients who had presented with an earlier stage of NSCLC or who did not initiate systemic therapy for NSCLC were excluded, as were those who had other cancer or who were participating in a clinical trial.

To gather the most recent data, eligible patients were identified starting at the end of the eligibility period (July 1, 2013) and working backwards in time. (In Germany, the eligibility period was extended to July 1,2014 , to recruit adequate patient numbers.) The index date for each patient was defined as the start date of first-line NSCLC therapy. The follow-up period was defined as being until the initiation of first medical record abstraction in the patient's country (in 2015 or 2016, depending on country), or until death, whichever occurred first [13]. 
The study protocol was approved by the appropriate institutional review board or independent ethics committee for each study site (Additional file 1). Informed consent was collected for patients from Italy and Spain who were alive at the time of data abstraction. In the other countries informed consent was not required for working with de-identified retrospective data.

\section{Outcome measures}

The key outcome measures were weekly HCRU rates and total HCRU, by line of therapy, related to management of Stage IIIB/IV NSCLC from the date of diagnosis until the end of follow-up for each patient. We included hospitalizations, emergency and outpatient visits, imaging tests, biopsy-related procedures, and biomarker tests. In addition, we examined hospitalizations and outpatient visits according to the results of predictive biomarker testing and whether testing was performed, with focus on testing for sensitizing EGFR mutations and $A L K$ gene rearrangements.

Hospitalization was defined as formal inpatient admittance to a hospital, either to a normal ward or intensive care unit, pursuant to an order for hospital admission by a physician or other qualified practitioner, for at least $24 \mathrm{~h}$. An emergency visit was defined as a visit to a hospital-based emergency department. An outpatient visit was defined as including outpatient hospital, officebased, and outpatient infusion therapy visits; officebased visits could be to a specialist or primary care provider.

\section{Statistical analyses}

This was an exploratory, descriptive study with no formal hypothesis testing. Data from patients' medical records were abstracted and reported using summary statistics by country. Data were not pooled across countries because of different clinical practices in each country. Available data were reported for key variables; missing data were not imputed.

All HCRU data were summarized over the entire course of the study. The summarized data were then averaged for each line of therapy (LOT) to calculate HCRU per 100 patient-weeks using the following formulas:

- Weeks of follow-up period $=$ (last visit/treatment stop date - index date +1$) / 7$

- Weeks of LOT $=$ (next LOT start date - LOT start date +1) / 7

- Weekly HCRU rate during each LOT per 100 patient-weeks $=100$ "summarized HCRU data for the LOT / weeks of LOT

All analyses were carried out using SAS version 9.4 (SAS Institute, Cary, NC, USA).

\section{Results}

\section{Patients and NSCLC therapy}

We studied a total of 1440 patients with advanced NSCLC in eight countries, including 174 patients in Italy, 202 patients in Spain, 139 patients in Germany, 208 patients in Australia, 175 patients in Japan, 150 patients in South Korea, 217 patients in Taiwan, and 175 patients in Brazil. The median age of patients in each country ranged from 63 to 70 years. The distributions of other demographic and clinical characteristics of patients in the individual countries were similar with the exception of Taiwan. In all countries except Taiwan, the majority of patients were male (from $53 \%$ to $77 \%$, but $48 \%$ male in Taiwan) and current or former smokers (65\% to $88 \%$, but $33 \%$ in Taiwan). Approximately threequarters of patients had NSCLC of nonsquamous histology, and over $80 \%$ in each country presented with stage IV disease, as previously reported [13, 14].

All patients received first-line systemic therapy per study enrollment criteria, and subsequently from $46 \%$ (Germany) to $71 \%$ (Taiwan) in each country received second-line therapy, and from 17\% (Brazil) to $42 \%$ (Taiwan) received third-line therapy. The majority of patients received platinum-based combinations for firstline therapy, most commonly carboplatin-paclitaxel, carboplatin-gemcitabine, or cisplatin-pemetrexed, except in Taiwan, where about half of patients received an EGFR TKI or ALK inhibitor. For second-line therapy, approximately half of patients received a single agent, most commonly docetaxel or pemetrexed, and approximately one-quarter received an EGFR TKI or ALK inhibitor. In general, treatment patterns by regimen category (platinum-based or non-platinum combination, single agent, EGFR/ALK TKI) varied only slightly by country, with the exception of first-line therapies in Taiwan $[13,14]$.

\section{Health care resource use by treatment line and regimen}

The proportion of patients who were hospitalized varied greatly by country (Tables 1 and 2): namely, during firstline, from $24 \%$ in Italy to $81 \%$ in Japan and, during second-line, from $22 \%$ in Italy to $84 \%$ in Japan were hospitalized at least once. In Germany during both firstand second-line therapy, three-quarters of patients had a recorded hospitalization. The proportions of patients hospitalized were generally similar by treatment regimen, except in Taiwan, where those who received platinum-based regimens were more likely to be hospitalized than those who received a single agent or an EGFR/ALK TKI (Tables 1 and 2). Instead, outpatient visits in association with first- and second-line therapy were recorded for most patients, including $86 \%$ or more in each country, except in Germany during first-line therapy (69\%; see Tables 1 and 2). 


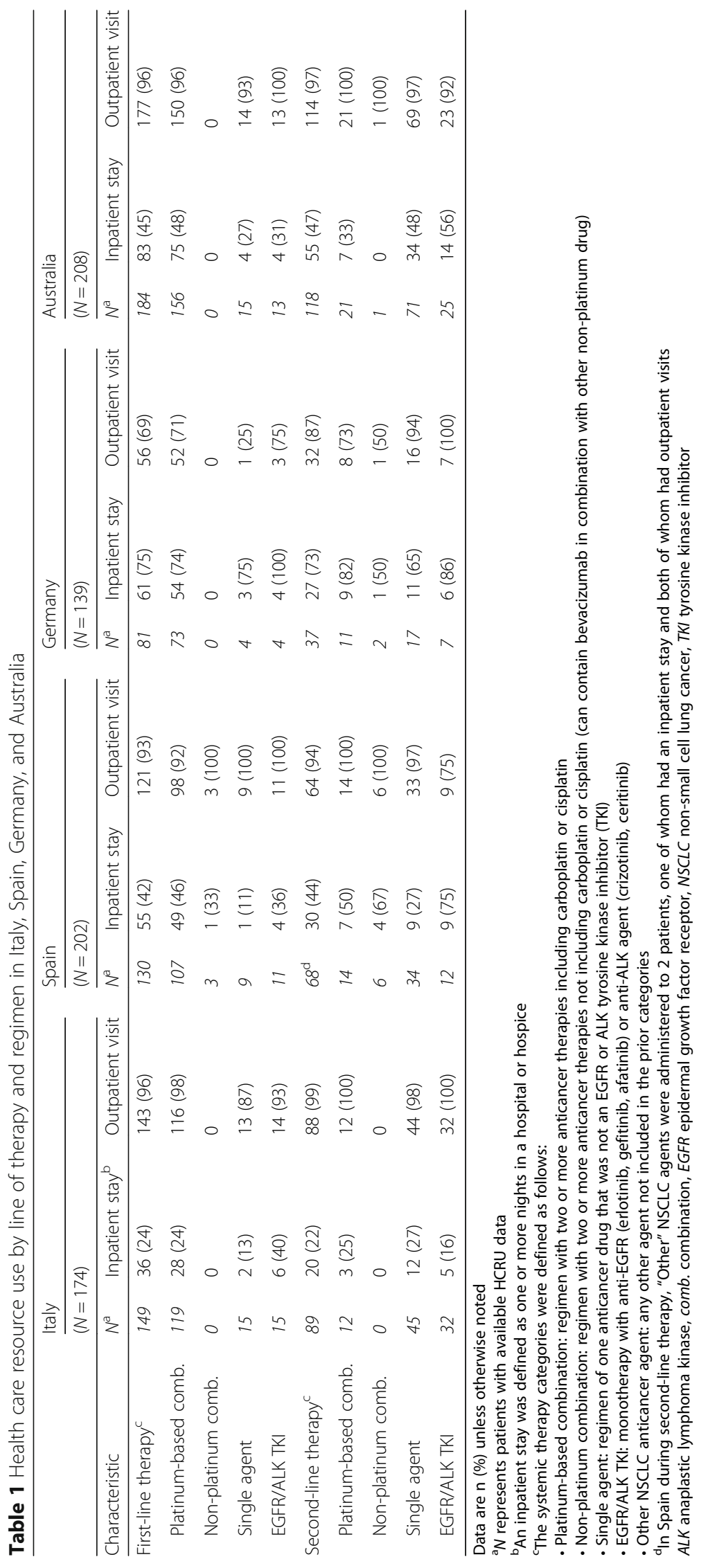




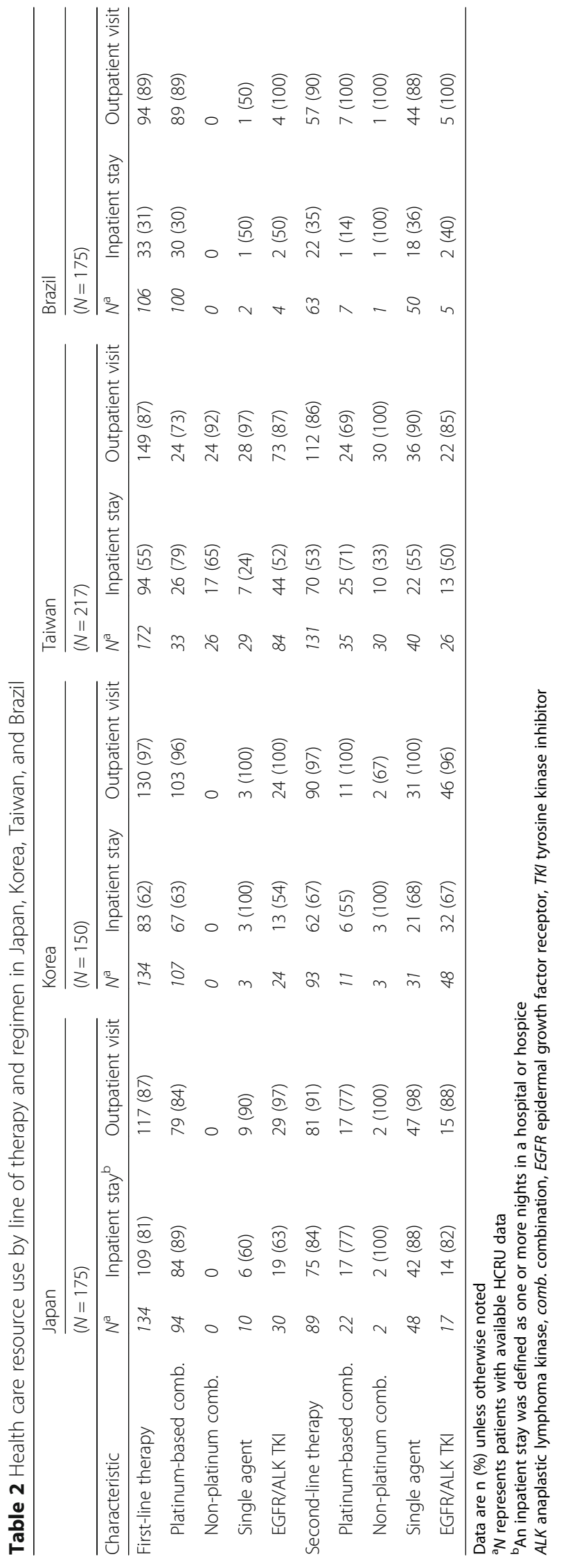


Table 3 provides further detail on hospitalizations, emergency visits, and outpatient visits by country, including further detail regarding previously published HCRU data from Japan [14]. During first-line therapy, the length of each hospitalization and the total days of hospitalization per patient were greatest in Japan, with a median of 15 days per hospitalization and a median of 36 total days of hospitalization. In the other countries the median lengths were from 3 to 7 days per hospitalization and 8-14 total days during first-line therapy (Table 3). A similar pattern persisted during second-line therapy and overall, depicted in Fig. 1. The number of hospitalizations per 100 patientweeks was highest in Korea in first-line and in Japan in second-line (Fig. 2). Admissions to hospice were few, recorded only in Spain, Australia, and Taiwan, with none in the other countries (Table 3).

The percentages of patients who were hospitalized as the result of a grade 3-5 adverse event during first-line therapy were highest in Australia, followed by Germany and Spain; during second-line, the percentages were highest in Spain, Australia, and Taiwan (Fig. 3). In some countries a higher percentage was hospitalized in second-line than first-line (Spain, Taiwan, and Italy); in the other countries the reverse was true. Overall, the percentages who were hospitalized in both first- and second-line therapy because of a grade 3-5 adverse event were lowest in Italy, in line with overall hospitalizations, and were also relatively low in Japan.

The frequency of emergency visits was low in Italy, Germany, and Japan and quite variable among the other countries (Table 3). However, in all countries a pattern of steadily increasing frequency from first- to third-line therapy was observed (Fig. 4).

The median number of outpatient visits per patient ranged from 4 (Germany) to 10 visits (Taiwan) during first-line therapy and from 3 (Germany and Brazil) to 10 visits (Taiwan) during second-line therapy. After accounting for follow-up time, the frequency of outpatient visits was greatest in Australia, Japan, and Korea during first-line (35-38 visits per 100 patient-weeks) and in Australia, Japan, Korea, and Taiwan during second-line (29-38 visits per 100 patient-weeks; Table 3, Fig. 5).

\section{Health care resource use by predictive biomarker status}

Tables 4 and 5 depict key HCRU variables according to predictive biomarker testing status and the results of testing. Only in Germany were the comparative findings consistent during first- and second-line therapy: namely, the patients with positive EGFR mutation or $A L K$ rearrangement status had longer median hospitalizations and more outpatient visits than those with negative or unknown test results.

In the other countries, there was no consistency in the pattern or differences in HCRU by biomarker status between the first and second lines of therapy. For example, during first-line therapy in Taiwan, which had the largest EGFR/ALK-positive cohort $(n=126)$, patients with positive $E G F R$ mutation or $A L K$ rearrangement status had longer median hospitalizations (7 vs. 3 days) and more outpatient visits (median, 12 vs. 6 visits) than those with negative or unknown test status, whereas during second-line therapy, the inverse was true (median 3 vs. 6 days in hospital and median of 9 vs. 12 outpatient visits, respectively; Table 5). In Korea, the median hospitalization lengths and median number of outpatient visits were the same or similar whether patients had positive or negative $E G F R / A L K$ mutation status or had not been tested.

\section{Health care resource use associated with procedures}

The median number of imaging tests per patient in association with first-line therapy was greatest in Japan (14 imaging tests), followed by Taiwan (median of 8) and Korea (median of 7; Table 6). The number of imaging tests per patient fell from first-line to second-line in all countries except Korea, where the median number of imaging tests per patient increased from 7 during first-line therapy to 8 during second-line therapy. Korea was also the only country in which positron emission tomography (PET) scans were frequently used: $70 \%$ of patients in Korea had a PET scan during first-line as compared with one-third or fewer patients in the other countries. Computed tomography (CT) scans were the most commonly employed imaging test in all countries, with $89 \%$ or more of patients in each country receiving a CT scan in association with first-line therapy (Table 6).

Biopsy-related procedures were employed almost exclusively in association with first-line therapy (Table 6). Similarly, predictive biomarker tests were run most frequently in association with first-line therapy, although some patients were tested for biomarkers in second-line, particularly in Australia. The most common biomarker testing in all countries was for EGFR mutations, with testing for $A L K$ rearrangements second in frequency. In Germany, Australia, and Korea, other tests such as for activating KRAS mutations were run for a substantial number of patients (Table 6).

\section{Discussion}

This retrospective observational study documented the health care resource use (HCRU) associated with first and subsequent lines of systemic therapy for advanced NSCLC for 1440 patients in eight countries. We found substantial regional variation in HCRU parameters, including the frequency and length of hospitalizations and the frequency of outpatient visits. Hospitalizations were most frequent in Japan, Korea, Germany, and Australia, relative to the other countries, and the length of each 
Lee et al. BMC Health Services Research (2018) 18:147

Page 7 of 16

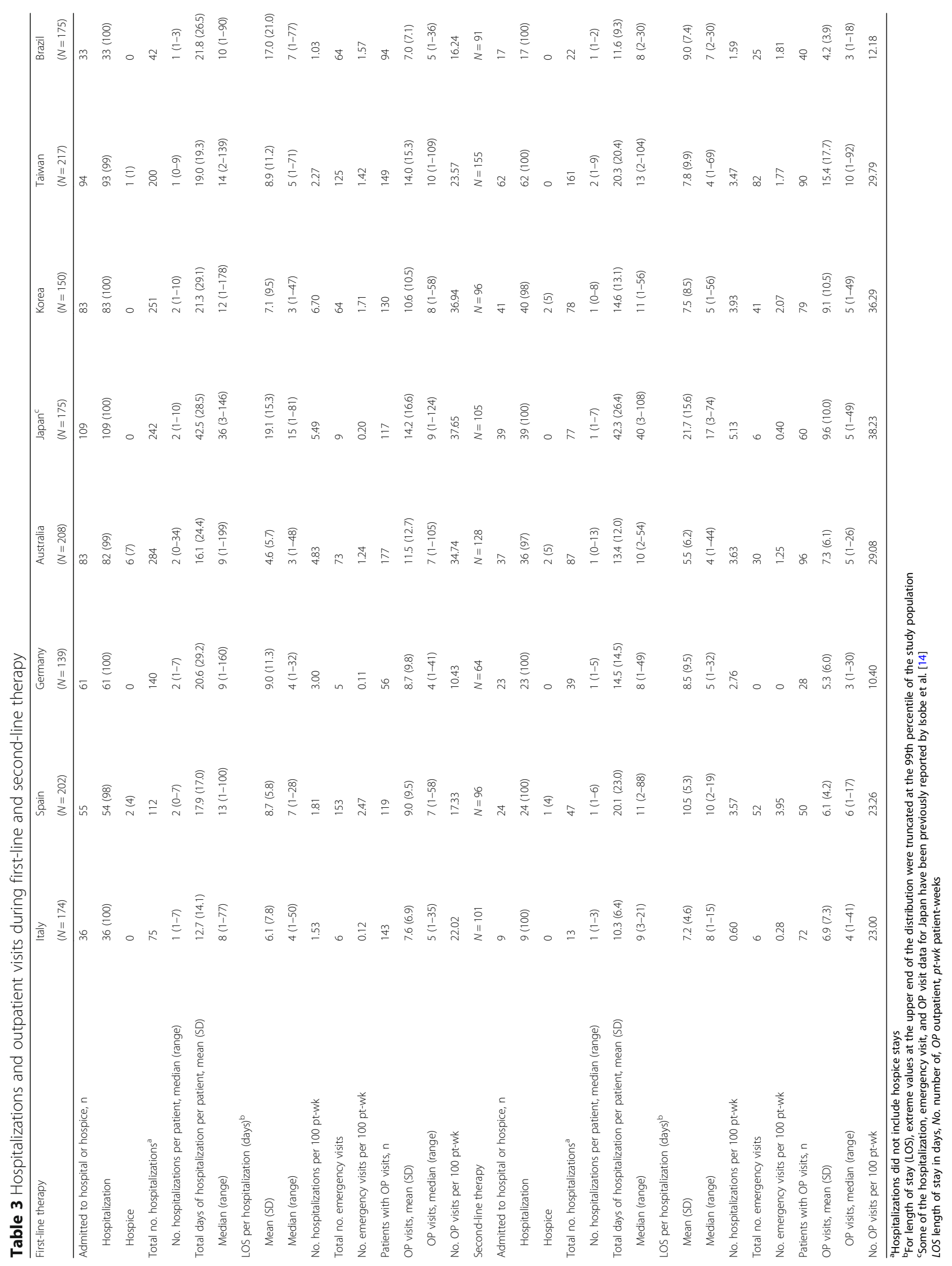




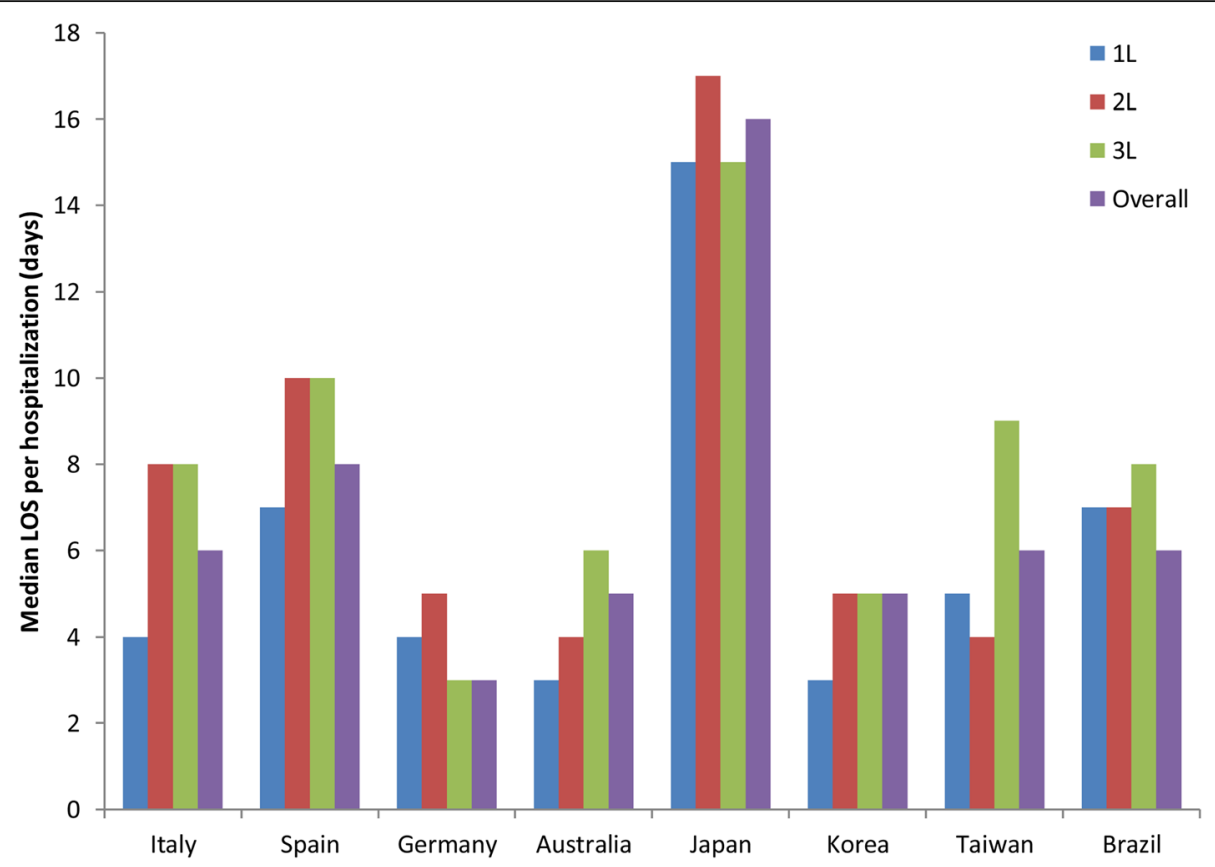

Fig. 1 Median length of stay (LOS) per hospitalization by line of therapy and overall in each country. 1L, 2L, 3L, during first-, second-, and thirdline therapy. Hospitalizations did not include hospice stays

hospital stay was longest in Japan during each line of therapy. While the frequency of emergency visits also varied among countries, we detected a consistent increase in the frequency of emergency visits from first- through thirdline therapy in each country. The outpatient setting was the most common setting of resource use. The evaluation of HCRU findings by predictive biomarker testing status identified no regular pattern, and the utilization and selection of imaging tests and predictive biomarker tests also varied among the countries. These findings suggest differing approaches to the clinical management of advanced NSCLC in the eight countries in this study.

There are few prior studies of real-world HCRU with which to compare our findings, in part because of differences in patient populations and in assessed variables. We conducted this study using one standard protocol in these eight countries in different regions in order to benchmark contemporary treatment practices and HCRU for patients

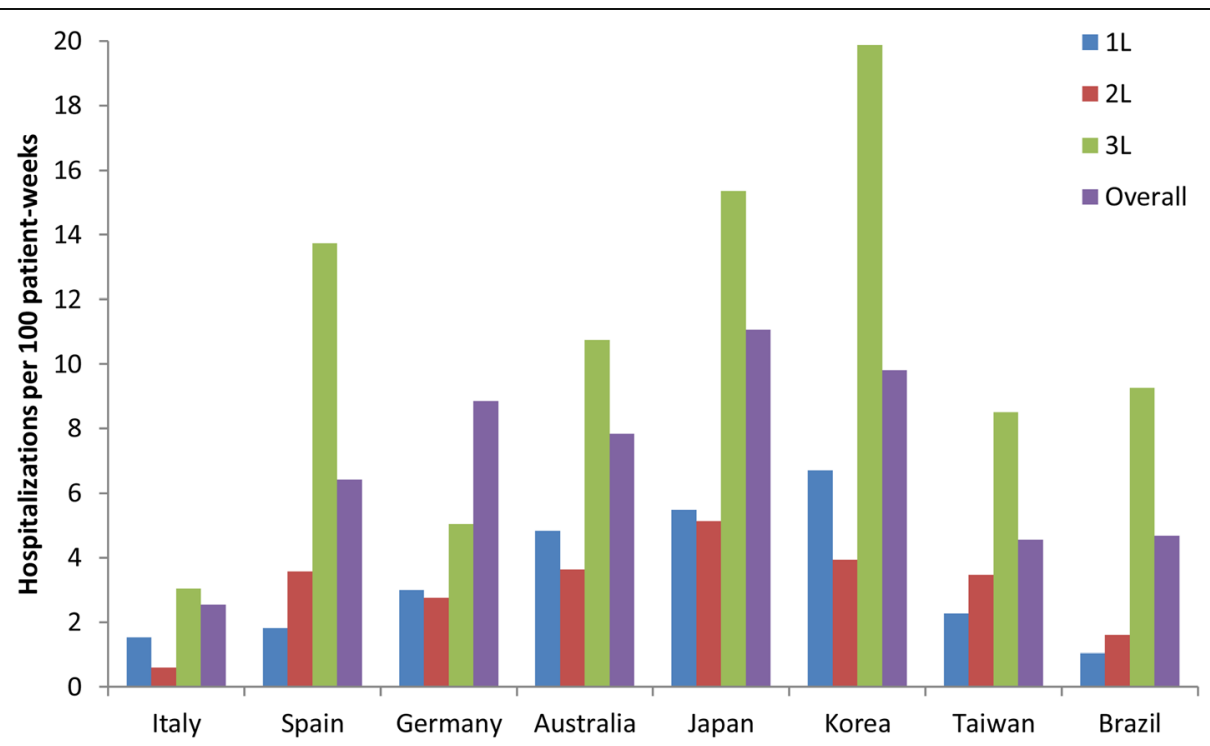

Fig. 2 Number of hospitalizations per 100 patient-weeks by line of therapy and overall in each country. 1L, 2L, 3L, during first-, second-, and third-line therapy. Hospitalizations did not include hospice stays 


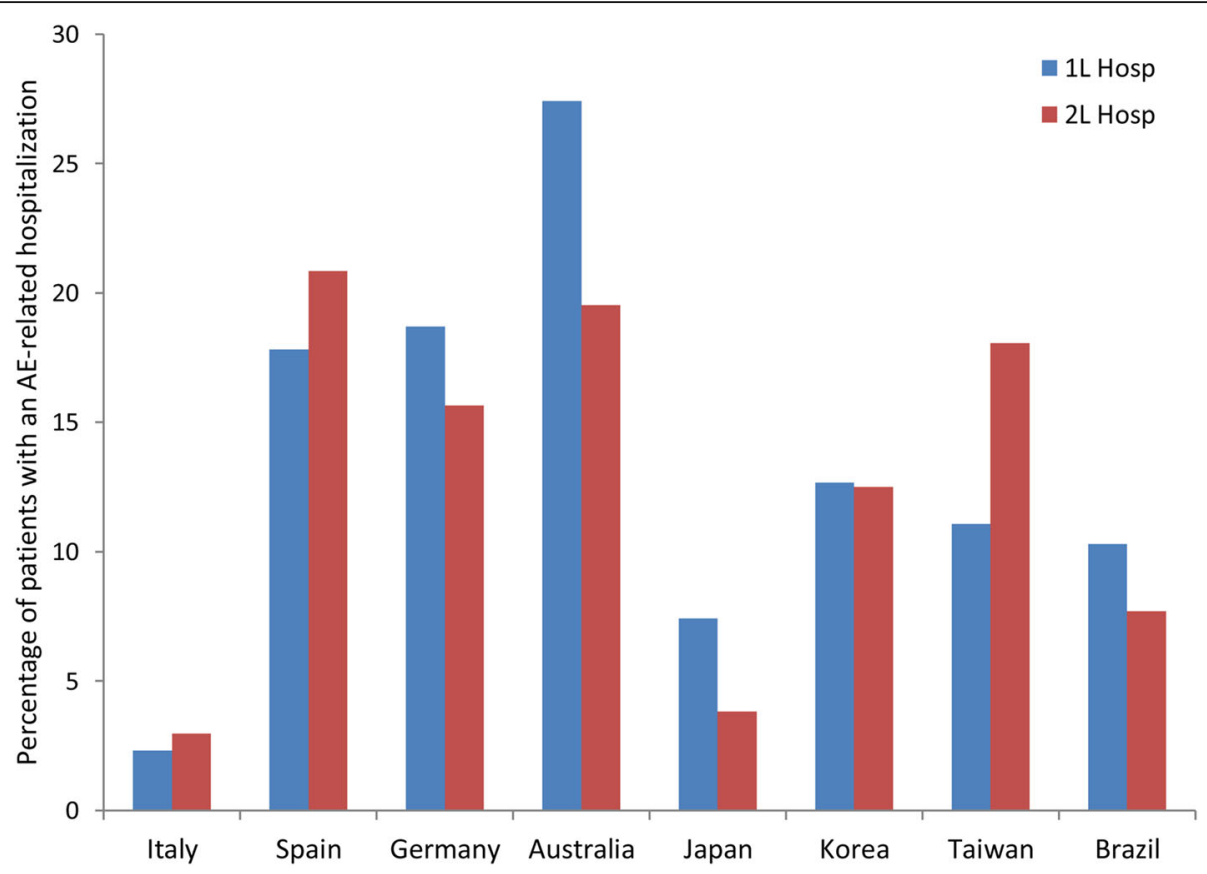

Fig. 3 Hospitalizations resulting from a grade 3-5 AE during first- and second-line therapy. 1L, 2L, first- and second-line therapy; $A E$, adverse event

receiving systemic treatment for advanced NSCLC. Nonetheless, our findings are purely descriptive and, because of the differences across countries in patient populations and health care settings, we cannot make definitive comparative statements. A 2013 review identified only two international studies of treatment patterns for NSCLC [10], neither of which reported HCRU findings $[15,16]$.
More recently, two multinational observational studies, both prospective, have been conducted in Europe, one limited to patients in 11 countries with advanced NSCLC prescribed first-line platinum-based chemotherapy (FRAME [17]) and the other including patients in 8 countries with all stages of NSCLC (EPICLIN-Lung [18]), thus neither directly comparable to our study. The HCRU

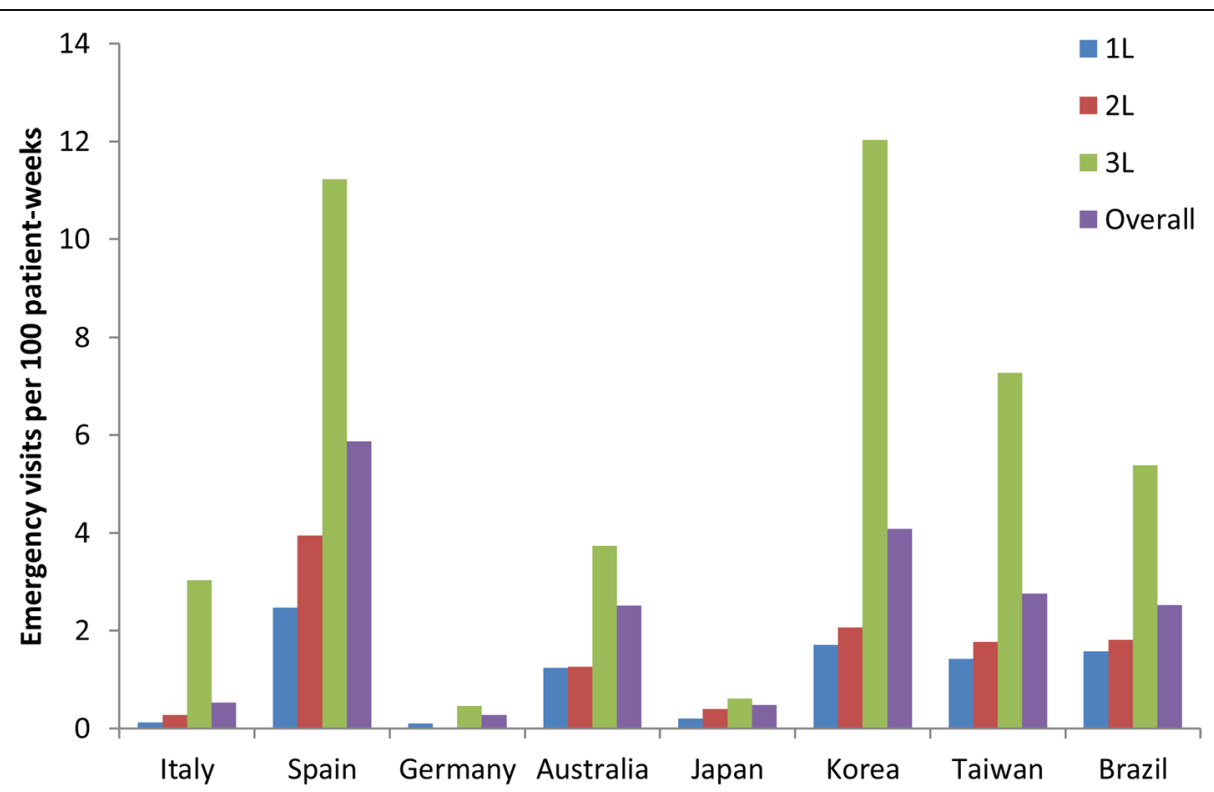

Fig. 4 Number of emergency visits per 100 patient-weeks by line of therapy and overall in each country. 1L, 2L, 3L, during first-, second-, and third-line therapy 


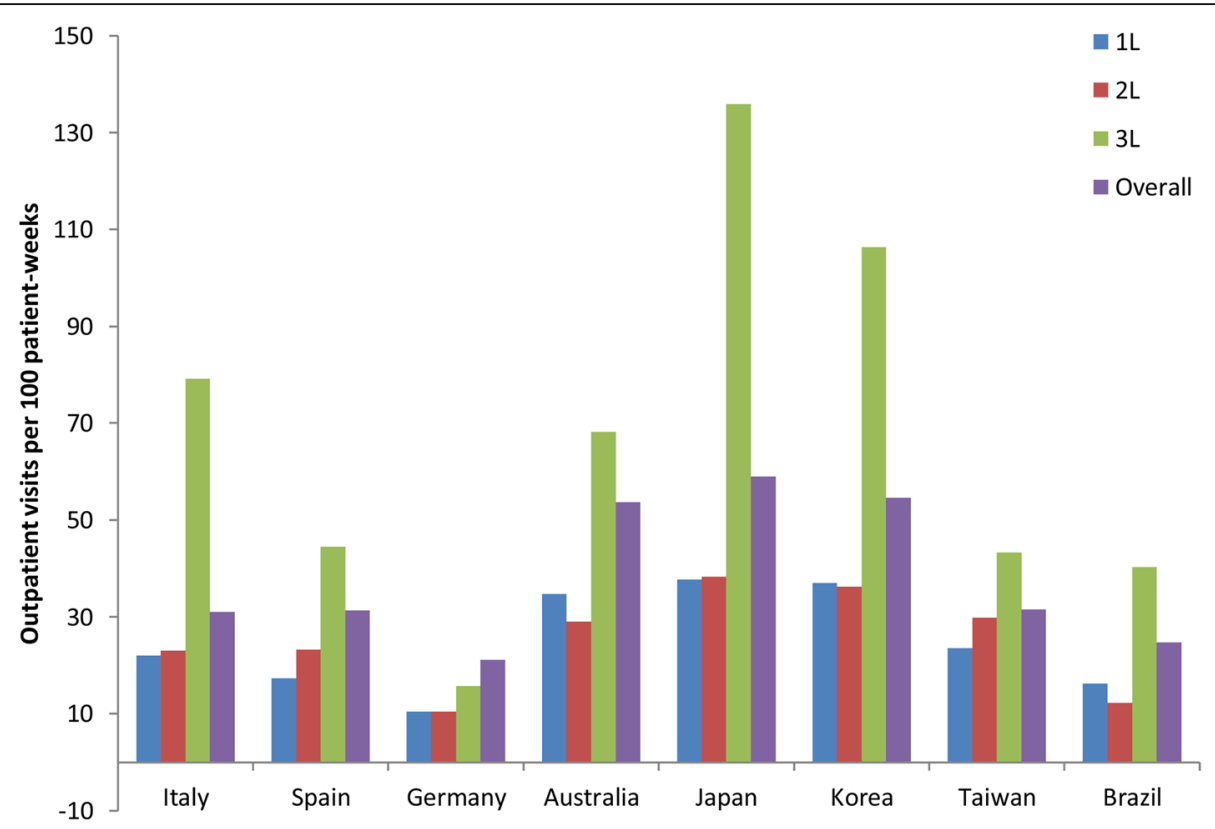

Fig. 5 Number of outpatient visits per 100 patient-weeks by line of therapy and overall in each country. 1L, 2L, 3L, during first-, second-, and third-line therapy

variables measured in these two studies also differed from those we used, and most results were pooled for the participating countries.

The few HCRU findings from FRAME and EPICLINLung that can be compared with ours relate to hospitalizations. In FRAME, 55\% of patients were hospitalized during first-line therapy, a percentage that ranged in the present study from $24 \%$ in Italy, to $42 \%$ in Spain, to $75 \%$ in Germany [17]. In EPICLIN-Lung, the total median numbers of hospital days for each patient were 9 and 10 days for stage IIIB and stage IV, respectively, as compared with medians of 8-13 days during first-line and 8-11 days during second-line therapy in the European countries in our study [18].

Our finding of the longest hospital stays being in Japan (mean of 19.1 and 21.7 days in first- and second-line, respectively) is consistent with results of the Organisation for Economic Cooperation and Development (OECD) summary of length of hospital stay for acute care (all causes) in 2015, in which the mean hospital stay for Japan (16.5 days) was the outlier among member countries, with Korea having the next longest mean stay (8.0 days) [19]. Few other studies have reported on hospitalization rates in individual countries. In one Australian medical center, patients with stage III and IV NSCLC spent a median of 13 and 18 total days in hospital, respectively [20], as compared with medians of 9 plus 10 total days during first- plus second-line, respectively, in our Australian cohort. In Canada, the frequency of hospitalizations was 7.0 per 100 patientweeks after the completion of chemotherapy [21], as compared with the overall frequency in our study of 2.5-11.1 per 100 patient-weeks, depending on country.

Patients with advanced NSCLC may be hospitalized for different reasons and at different times during the course of their illness. Reasons for hospitalization include initial and subsequent diagnostic testing, anticancer therapy administration, patient monitoring, adverse event management, and provision of supportive care. The decision to hospitalize a patient in any given situation is likely influenced by preferred clinical practices, which could explain the variability in frequency and length of hospitalizations found in this study. The pattern of hospitalizations resulting from grade $3-5$ adverse events did not follow the overall pattern of hospitalizations (for any reason), further suggesting that clinical practices varied among the countries. The percentages of patients who were hospitalized because of a grade 3-5 adverse event during first-line therapy were highest in Australia and lowest in Italy, in line with overall hospitalizations, but were also relatively low in Japan, where overall hospitalizations were most frequent. Clinical trials rarely report information on hospitalizations, and the results of one recent review concluded that real-world patients undergoing chemotherapy for metastatic NSCLC have significantly higher rates of hospitalization than those in clinical trials receiving similar therapy [11], further supporting the need for realworld studies such as this one.

In European countries, lung cancer had the greatest associated economic burden of four common cancers (including also breast, colorectal, and prostate cancer) [22]. We did not evaluate the costs associated with 


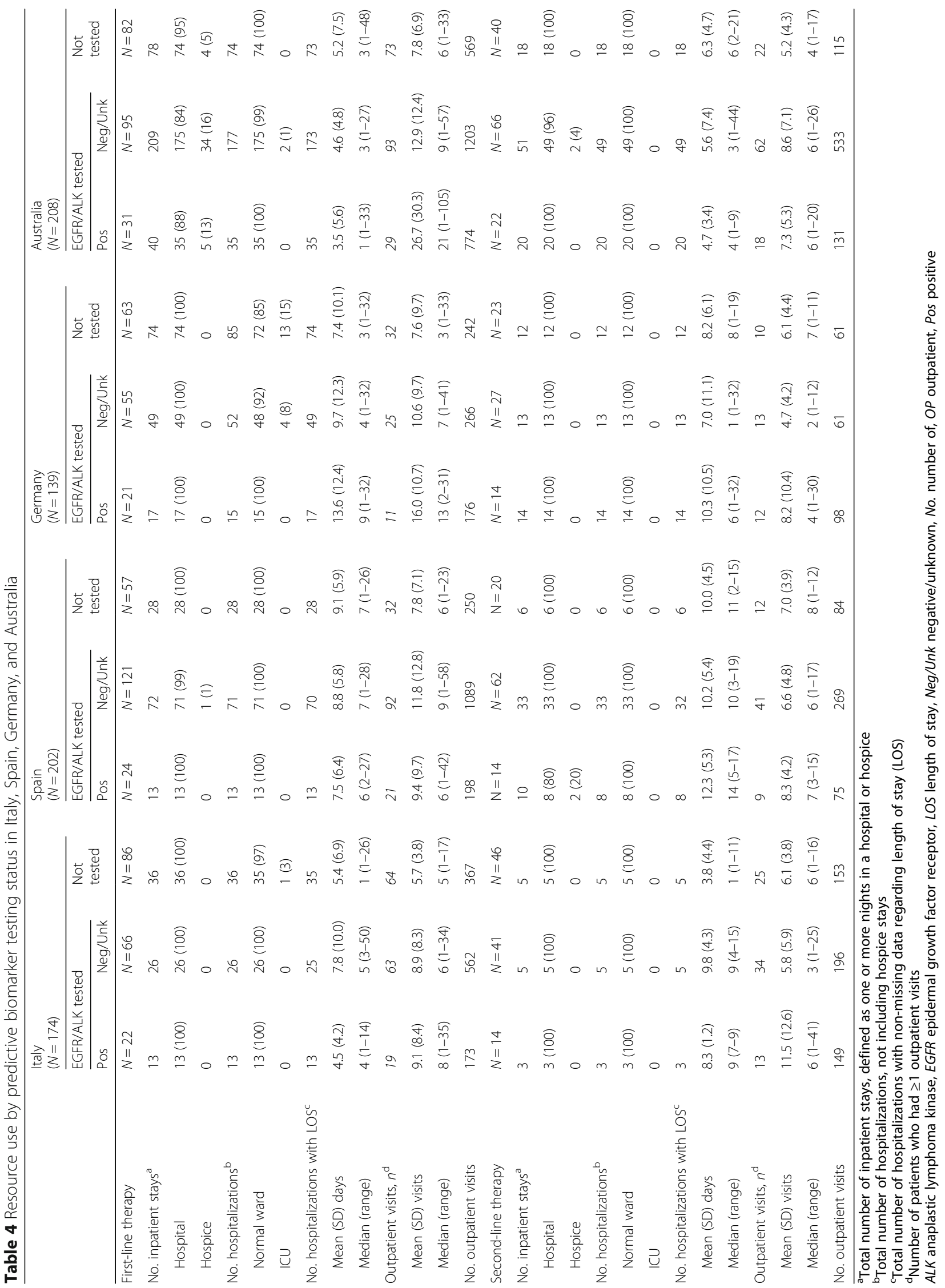




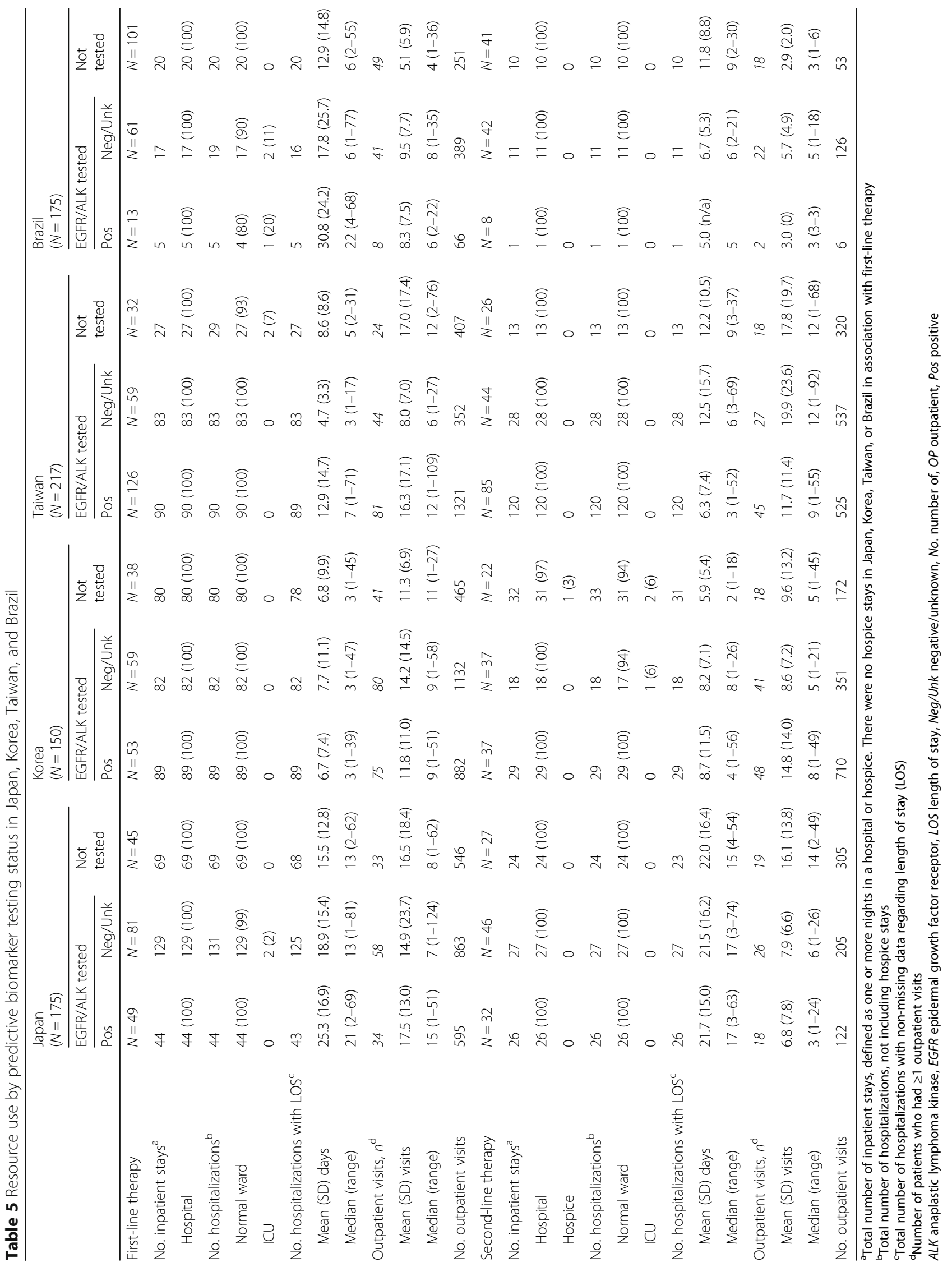




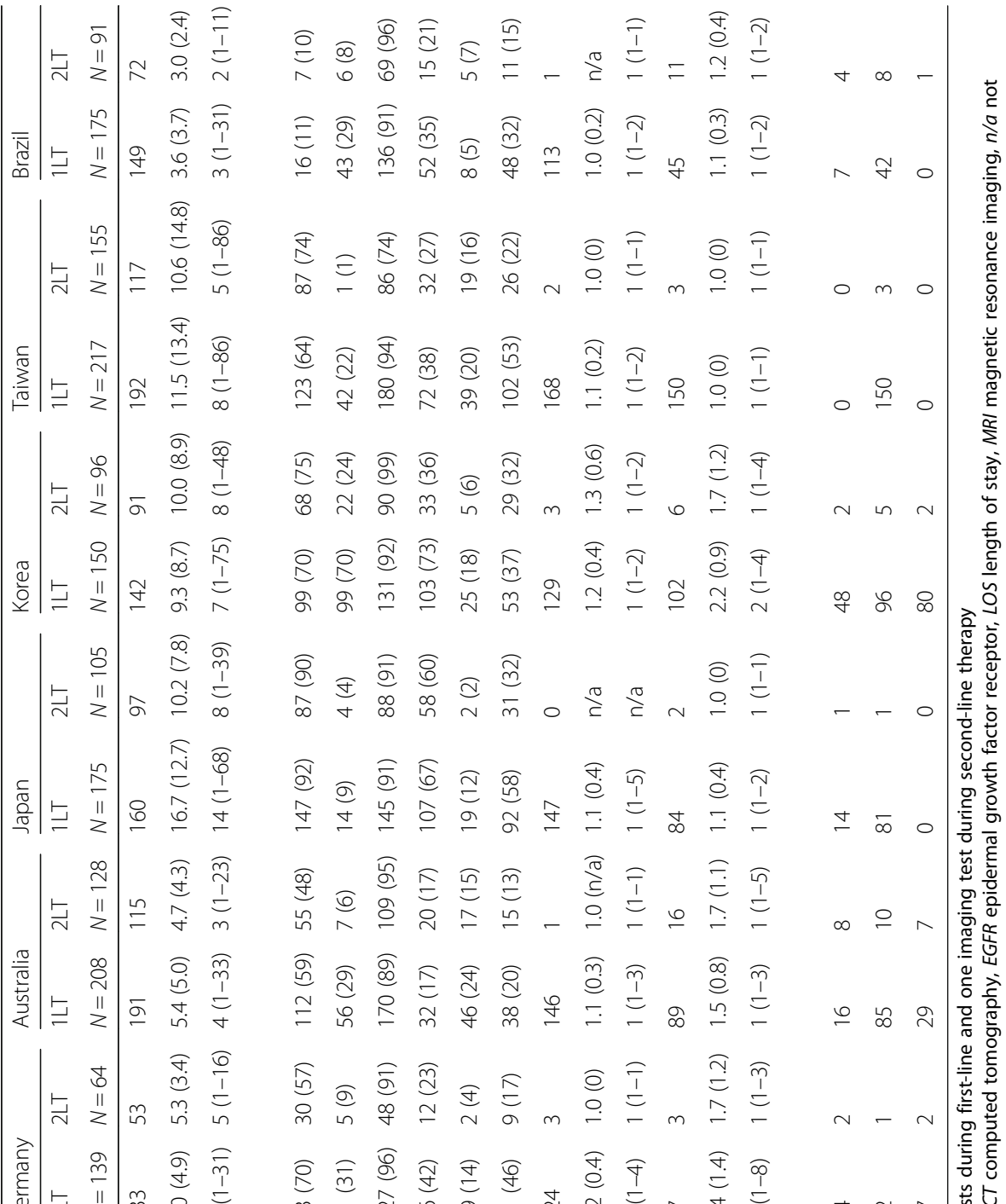


HCRU in the present study; however, hospitalizations are acknowledged as being a major cost driver for patients with advanced NSCLC in studies in several countries [20, 21, 23-26]. Other important factors driving costs include fees for medical specialists in the Netherlands [23], physicians' fees [25] and outpatient and inpatient medical services [27] in the United States, and drug costs in China, where drug consumption is a means of paying for physicians' work [28].

One of the strengths of our study is that we were able to examine HCRU findings by predictive biomarker testing status. We found no regular pattern of HCRU in association with first- or second-line therapy according to whether patients were tested for EGFR mutation or $A L K$ rearrangement, nor whether the results were positive, negative, or unknown. In Taiwan, where testing for EGFR mutation was most frequent and first-line therapy with an EGFR TKI was most common, the relatively large cohort of patients with positive EGFR/ALK status experienced longer hospitalizations and more outpatient visits during first-line therapy but shorter hospitalizations and fewer outpatient visits during second-line therapy than patients whose EGFR/ALK test results were negative or unknown. However, the proportion of patients who received EGFR/ALK TKIs was much lower in second-line as compared with first-line therapy in Taiwan, hence the EGFR/ALK status may not have had an impact on resource use. These findings require further investigation. Results of an earlier small study in Korea suggested that the mean monthly costs were lower for patients with EGFR-mutation positive status who received targeted treatment for advanced NSCLC as compared with patients who had wild-type EGFR status [29].

The frequency of emergency visits increased in all countries in this study over time, from first- to third-line therapy. Lung cancer, as compared with other cancers, has been identified in a recent systematic review as being a risk factor for emergency department attendance in the last month of life [30]. The authors of this review did not offer an explanation for their finding but note that future investigations are needed of psychosocial factors and patient and caregiver preferences for using emergency care services. The use of health care resources is driven by clinicians' and patients' preferences, and of course by patients' performance status and the presence or absence of metastases and comorbidities. As previously reported, hospitalizations and HCRU increase with skeletal metastases [31-33], and patients with brain metastases have greater HCRU and incur greater health care costs [34-36].

Regional and within-country variations in NSCLC treatment patterns and HCRU parameters have been recorded also in prior studies. We can speculate that these differences are at least in part attributable to variability in NSCLC management guidelines and in health care systems, including reimbursement policies and insurance options. The median age of patients varied among countries from 63 to 70 years, and the Taiwanese study population was notable in having the lowest proportion of men and current or former smokers, perhaps because of variability in attendance at study sites or possible selection bias. Tsukada and coworkers [37] studied the characteristics of cancer populations among different types of hospitals in Japan, and they found higher proportions of early-stage cancer and younger patients at highervolume hospitals than at lower-volume hospitals. Substantial variations in patterns of care for NSCLC among the different hospital types were recorded in their study in Japan [37], as well as in prior observational studies in Taiwan [38] and the Netherlands [39, 40]. Our study included a mix of hospital types. All sites in Australia and Taiwan, and the majority of sites in Spain (10 of 13 [10/ $13])$ and Korea $(2 / 3)$ were centers affiliated with an academic institution; in Italy, Germany, and Japan the inverse was true, as $3 / 9,3 / 10$, and $1 / 5$ sites, respectively, were academic; and in Brazil, study sites were a mix of academic $(n=6)$ and non-academic $(n=5)$ [13]. Therefore, within-country variation in patterns of care among different sites in our study is also possible.

A limitation of this study is that we were not able to link patient and NSCLC clinical characteristics with the quantity of HCRU. Other study limitations are those inherent to observational studies using medical records, including the afore-mentioned potential for selection bias in addition to the possibility of missing and/or incorrectly recorded data. A standardized approach was used to collect study data at each study site using the eCRFs, and we provided training to the site coordinators to promote consistent recording among the study sites; however, the potential for inconsistent recording remains a study limitation.

Strengths of this descriptive study include the large patient population and the capture of real-world resource use for systemic therapy of advanced NSCLC in eight different countries. We evaluated and report HCRU according to line of therapy, including three lines of therapy, and overall, in addition to biomarker testing status, with a minimum of 12 months' follow-up for each patient. The diagnosis of advanced NSCLC occurred from the start of 2011 to mid-2014; thus, the data illustrate relatively recent clinical practices for the diagnosis and treatment of advanced NSCLC.

Further research is needed to evaluate HCRU in countries not included in this study, as well as to update and expand our findings in the countries studied, to inform the administration of health care resources. A contemporary comparison of HCRU for NSCLC among 
different hospital types within each country is an important topic for future study that was beyond the scope of the present study. Another important topic for future study is to identify the proportion of patients who do not receive treatment for advanced NSCLC and the reasons why treatment is not provided to (or chosen by) patients.

\section{Conclusions}

In 2013, De Geer et al. [10] noted the scarcity of studies of real-world treatment patterns and HCRU for advanced NSCLC. This remains largely true today. The prevalence of lung cancer has decreased slightly among men in some countries but is projected to continue rising among both men and overall in other countries, particularly where tobacco smoking remains common [1]. Lung cancer will remain a major health care burden for the foreseeable future. We hope that the data provided here will be useful for policy- and decisionmakers in each country to determine future resource allocation. More studies are needed of real-world clinical practices and HCRU for advanced NSCLC to benchmark current practices as a baseline in light of the everincreasing availability of new therapies, associated resource-intensive health care needs, and increasing budgetary pressures on health care systems. Comparative findings and an understanding of country-specific clinical practices are essential to identify areas of need and to guide future resource allocation for patients with advanced NSCLC.

\section{Additional file}

Additional file 1: Ethics Committee Names and Locations. (XLSX 13 kb)

\section{Abbreviations}

ALK: Anaplastic lymphoma kinase; CT: Computed tomography; eCRF: Electronic case report forms; EGFR: Epidermal growth factor receptor; HCRU: Health care resource use; LOT: Line of therapy; NSCLC: Non-small cell lung cancer; PD-1: Programmed death-1; PET: Positron emission tomography; TKl: Tyrosine kinase inhibitor

\section{Acknowledgements}

We thank all the investigators who participated in this study and QuintilesIMS (Cambridge, MA, USA) for data acquisition and statistical support. In addition, we thank Qian Xia and Mary Anne Rutkowski (Merck \& Co., Inc., Kenilworth, NJ, USA) for statistical programming support and Reshma Shinde and Teesta Banerjee (Merck \& Co., Inc., Kenilworth, NJ, USA) for editorial and administrative support. Medical writing and editorial assistance was provided by Elizabeth V. Hillyer, DVM. This assistance was funded by Merck Sharp \& Dohme Corp., a subsidiary of Merck \& Co., Inc., Kenilworth, NJ, USA.

\section{Funding}

This study was sponsored by Merck \& Co., Inc., Kenilworth, NJ, USA. The funder of the study was responsible for the study design and funded the collection and analysis of the data. All authors, including those employed by Merck, participated in the data interpretation and writing of the manuscript.

\section{Availability of data and materials}

The data used in this study are abstracted from individual patient records in eight countries and are not publicly available.

\section{Authors' contributions}

AA and SK conceived and designed the study. DHL, HI, HW, SBA, PP, FdM, $\mathrm{JdC}$ contributed to protocol review and recruited patients. $X \mathrm{C}$ analyzed the data. All authors (DHL, HI, HW, SBA, PP, FdM, MH, AA, SK, XC, ND, A-MW, JdC) contributed to the interpretation of findings and the critical review and revision of the manuscript. All authors read and approved the final manuscript.

\section{Ethics approval and consent to participate}

The study protocol was approved by the appropriate institutional review board or independent ethics committee for each study site. Informed consent was collected for patients from Italy and Spain who were alive at the time of data abstraction; an informed consent form was not required from deceased patients' next of kin. In the other countries, informed consent was not required for working with the de-identified retrospective data used in the study. The names and locations of the ethics committees are summarized in the Additional file 1.

\section{Consent for publication}

Not applicable.

\section{Competing interests}

Dae Ho Lee reports honoraria from AstraZeneca, Boehringer-Ingelheim, Bristol-Myers Squibb, CJ Healthcare, Eli Lilly, Jansen, Merck, MSD, Mundipharma, Novartis, Ono, Pfizer, Roche, Samyang Biopharm, and ST Cube for participating in advisory boards; and receipt of consulting fees from the Ministry of Food and Drug Safety (MFDS), Korea, and Health Insurance Review and Assessment Service (HIRA), Korea.

Hiroshi Isobe has received payment for speaking engagements from AstraZeneca, Bristol-Myers Squibb, Kyowa Hakko Kirin, Chugai, Boehringer Ingelheim, Ono Pharmaceutical Co. Ltd., and Eli Lilly Japan, K.K. Hubert Wirtz reports lecture fees from AstraZeneca, Roche, and Boeringer Ingelheim. Filippo de Marinis reports advisory role for Roche, BMS, and AstraZeneca.

Ann-Marie Woodgate and Nello Donnini are employees of Merck Sharp \& Dohme.

Min Huang, Ashwini Arunachalam, and Smita Kothari are employees of Merck \& Co., Inc..

Xiting Cao, is an employee of Merck \& Co., Inc. and owns stock options from Merck.

The other authors have no competing of interest to declare.

\section{Publisher's Note}

Springer Nature remains neutral with regard to jurisdictional claims in published maps and institutional affiliations.

\section{Author details \\ 'Asan Medical Center, Seoul, Republic of Korea. ${ }^{2}$ KKR Sapporo Medical Center, Sapporo, Japan. ${ }^{3}$ University of Leipzig, Leipzig, Germany. ${ }^{4}$ Oncology Services, Hospital Evangelico, Cachoeiro de Itapemirim, Brazil. ${ }^{5}$ Cancer Services, Box Hill Hospital, and Monash University, Victoria, Australia. ${ }^{6}$ Thoracic Oncology Division, European Institute of Oncology (IEO), Milan, Italy. ${ }^{7}$ Center for Observational and Real World Evidence (CORE), Merck \& Co., Inc.. North Wales, PA, USA. ${ }^{8}$ Center for Observational and Real World Evidence (CORE), Merck \& Co., Inc., 2000 Galloping Hill Road, Kenilworth, NJ 07033, USA. ${ }^{9}$ MSD Italia, Rome, Italy. ${ }^{10}$ MSD Australia \& New Zealand, Sydney, Australia. ${ }^{11}$ Medical Oncology Service, Hospital Universitario La Paz (IDIPAZ),} Madrid, Spain

Received: 6 April 2017 Accepted: 19 February 2018

Published online: 01 March 2018

\section{References}

1. Cheng TY, Cramb SM, Baade PD, Youlden DR, Nwogu C, Reid ME. The international epidemiology of lung cancer: latest trends, disparities, and tumor characteristics. J Thorac Oncol. 2016;11(10):1653-71.

2. Herbst RS, Heymach JV, Lippman SM. Lung cancer. N Engl J Med. 2008; 359(13):1367-80. 
3. Goldstraw P, Ball D, Jett JR, Le Chevalier T, Lim E, Nicholson AG, et al. Non-small-cell lung cancer. Lancet. 2011;378(9804):1727-40.

4. American Cancer Society. Key statistics for lung cancer. https:/www.cancer. org/cancer/non-small-cell-lung-cancer/about/key-statistics.html. Accessed 24 Feb 2018.

5. Morgensztern D, Ng SH, Gao F, Govindan R. Trends in stage distribution for patients with non-small cell lung cancer: a National Cancer Database survey. J Thorac Oncol. 2010;5(1):29-33.

6. Reck M, Heigener DF, Mok T, Soria JC, Rabe KF. Management of non-smallcell lung cancer: recent developments. Lancet. 2013;382(9893):709-19.

7. Reck M, Paz-Ares L. Immunologic checkpoint blockade in lung cancer. Semin Oncol. 2015;42(3):402-17.

8. Langer $\mathrm{CJ}$. Emerging immunotherapies in the treatment of non-small cell lung cancer (NSCLC): the role of immune checkpoint inhibitors. Am I Clin Oncol. 2015:38(4):422-30.

9. Morgensztern D, Herbst RS. Nivolumab and pembrolizumab for non-small cell lung cancer. Clin Cancer Res. 2016;22(15):3713-7.

10. De Geer A, Eriksson J, Finnern HW. A cross-country review of data collected on non-small cell lung cancer (NSCLC) patients in cancer registries, databases, retrospective and non-randomized prospective studies. J Med Econ. 2013;16(1):134-49.

11. Prince RM, Atenafu EG, Krzyzanowska MK. Hospitalizations during systemic therapy for metastatic lung cancer: a systematic review of real world vs clinical trial outcomes. JAMA Oncol. 2015;1(9):1333-9.

12. Garrison LP Jr, Neumann PJ, Erickson P, Marshall D, Mullins CD. Using realworld data for coverage and payment decisions: the ISPOR real-world data task force report. Value Health. 2007;10(5):326-35.

13. de Castro J, Tagliaferri P, de Lima VCC, Ng S, Thomas M, Arunachalam A, et al. Systemic therapy treatment patterns in patients with advanced nonsmall cell lung cancer (NSCLC): PlvOTAL study. Eur J Cancer Care (Engl). 2017;26:e12734. https://doi.org/10.1111/ecc.12734.

14. Isobe H, Mori K, Minato K, Katsura H, Taniquchi K, Arunachalam A, et al. Real-world practice patterns for patients with advanced non-small cell lung cancer: multicenter retrospective cohort study in Japan. Lung Cancer (Auckl). 2017;8:191-206.

15. Bischoff HG, van den Borne B, Pimentel FL, Arellano J, Langer F, Leschinger $\mathrm{Ml}$, et al. Observation of the treatment and outcomes of patients receiving chemotherapy for advanced NSCLC in Europe (ACTION study). Curr Med Res Opin. 2010;26(6):1461-70.

16. Vergnenegre A, Smit EF, Toy E, Parente B, Schmitz S, Kraaij K, et al. Secondline therapy for non-small cell lung cancer in clinical practice: final results and treatment pathways from the SELECTTION observational study. Curr Med Res Opin. 2012;28(8):1253-62.

17. Moro-Sibilot D, Smit E, de Castro Carpeno J, Lesniewski-Kmak K, Aerts J, Villatoro R, et al. Outcomes and resource use of non-small cell lung cancer (NSCLC) patients treated with first-line platinum-based chemotherapy across Europe: FRAME prospective observational study. Lung Cancer. 2015;88(2): 215-22.

18. Vergnenegre A, Carrato A, Thomas M, Jernigan C, Medina J, Cruciani G. Real-world healthcare resource utilization in a European non-small cell lung cancer population: the EPICLIN-lung study. Curr Med Res Opin. 2014;30(3): 463-70.

19. Organization for Economic Cooperation and Development (OECD). Length of hospital stay (indicator): Acute care, days, 2015. https://doi.org/10.1787/ 8dda6b7a-en. Accessed 24 Feb 2018.

20. Kang S, Koh ES, Vinod SK, Jalaludin B. Cost analysis of lung cancer management in south western Sydney. J Med Imaging Radiat Oncol. 2012;56(2):235-41.

21. Navaratnam S, Kliewer EV, Butler J, Demers AA, Musto G, Badiani K. Population-based patterns and cost of management of metastatic nonsmall cell lung cancer after completion of chemotherapy until death. Lung Cancer. 2010;70(1):110-5.

22. Luengo-Fernandez R, Leal J, Gray A, Sullivan R. Economic burden of cancer across the European Union: a population-based cost analysis. Lancet Oncol. 2013:14(12):1165-74

23. van der Linden N, Bongers ML, Coupe VM, Smit EF, Groen HJ, Welling A, et al. Costs of non-small cell lung cancer in the Netherlands. Lung Cancer. 2016;91:79-88

24. Pompen M, Gok M, Novak A, van Wuijtswinkel R, Biesma B, Schramel F, et al. Direct costs associated with the disease management of patients with unresectable advanced non-small-cell lung cancer in The Netherlands. Lung Cancer. 2009;64(1):110-6.
25. Lang K, Marciniak MD, Faries D, Stokes M, Buesching D, Earle C, et al. Costs of first-line doublet chemotherapy and lifetime medical care in advanced non-small-cell lung cancer in the United States. Value Health. 2009:12(4):481-8.

26. Nieder C, Tollali T, Norum J, Pawinski A, Bremnes RM. A population-based study of the pattern of terminal care and hospital death in patients with non-small cell lung cancer. Anticancer Res. 2012;32(1):189-94.

27. Vera-Llonch M, Weycker D, Glass A, Gao S, Borker R, Barber B, et al. Healthcare costs in patients with metastatic lung cancer receiving chemotherapy. BMC Health Serv Res. 2011;11:305.

28. Shi J, Zhu J. Health resource utilization in patients with advanced non-small cell lung cancer receiving chemotherapy in China. Clin Drug Investig. 2016; 36(1):77-86.

29. Lee JK, Kim DW, Keam B, Kim TM, Lee SH, Kim YJ, et al. Impact of molecularly targeted treatment on direct medical costs in patients with advanced non-small cell lung cancer. Cancer Res Treat. 2015;47(2):182-8.

30. Henson LA, Gao W, Higginson IJ, Smith M, Davies JM, Ellis-Smith C, et al. Emergency department attendance by patients with cancer in their last month of life: a systematic review and meta-analysis. J Clin Oncol. 2015; 33(4):370-6

31. Hoefeler H, Duran I, Hechmati G, Garzon Rodriguez C, Luftner D, Ashcroft J, et al. Health resource utilization associated with skeletal-related events in patients with bone metastases: results from a multinational retrospective prospective observational study - a cohort from 4 European countries. J Bone Oncol. 2014;3(2):40-8.

32. Lorusso V, Duran I, Garzon-Rodriquez C, Luftner D, Bahl A, Ashcroft J, et al. Health resource utilisation associated with skeletal-related events in European patients with lung cancer: alpha subgroup analysis from a prospective multinational study. Mol Clin Oncol. 2014;2(5):701-8.

33. Skov Dalgaard K, Gammelager H, Svaerke C, Kurics T, Cetin K, Christiansen CF. Hospital use among patients with lung cancer complicated by bone metastases and skeletal-related events: a population-based cohort study in Denmark. Clin Epidemiol. 2015;7:363-8.

34. Ray S, Dacosta-Byfield S, Ganguli A, Bonthapally V, Teitelbaum A. Comparative analysis of survival, treatment, cost and resource use among patients newly diagnosed with brain metastasis by initial primary cancer. J Neuro-Oncol. 2013:114(1):117-25.

35. Guerin A, Sasane M, Zhang J, Culver KW, Dea K, Nitulescu R, et al. Brain metastases in patients with ALK+ non-small cell lung cancer: clinical symptoms, treatment patterns and economic burden. J Med Econ. 2015; 18(4):312-22.

36. Peters $\mathrm{S}$, Bexelius $\mathrm{C}$, Munk V, Leighl N. The impact of brain metastasis on quality of life, resource utilization and survival in patients with non-smallcell lung cancer. Cancer Treat Rev. 2016;45:139-62.

37. Tsukada Y, Nakamura F, Iwamoto M, Nishimoto H, Emori Y, Terahara A, et al. Are hospitals in Japan with larger patient volume treating younger and earlier-stage cancer patients? An analysis of hospital-based cancer registry data in Japan. Jpn J Clin Oncol. 2015:45(8):719-26.

38. Chien CR, Tsai CM, Tang ST, Chung KP, Chiu CH, Lai MS. Quality of care for lung cancer in Taiwan: a pattern of care based on core measures in the Taiwan cancer database registry. J Formos Med Assoc. 2008;107(8):635-43.

39. Wouters MW, Siesling S, Jansen-Landheer ML, Elferink MA, Belderbos J, Coebergh JW, et al. Variation in treatment and outcome in patients with non-small cell lung cancer by region, hospital type and volume in the Netherlands. Eur J Surg Oncol. 2010;36(Suppl 1):S83-92.

40. van der Linden N, Bongers ML, Coupe VMH, Smit EF, Groen HJM, Welling A, et al. Treatment patterns and differences in survival of non-small cell lung cancer patients between academic and non-academic hospitals in the Netherlands. Clin Lung Cancer. 2017;18(5):e341-e7. 